\title{
Education \& Outreach Programs at KEK
}

\author{
Shota Takahashi* \\ KEK IPNS \\ E-mail: shotakahepost.kek.jp
}

KEK is one of the world's major particle physics research organizations located in Japan. Our mission in research is to unveil mysteries of the Universe, shedding a light to expand the knowledge of human beings. However, sharing these knowledge is also as important as accomplishing our missions. For that purpose, we have educational programs such as science camps, masterclasses, hands-on experiences for students from elementary school to undergraduates. We also organise science cafe, public lectures, open campus, photo exhibition, and concert for general public. In this proceeding, three out of many education \& outreach programs at KEK are described. We would like to introduce these programs and discuss how to make physics, especially high energy physics, more popular in Japan.

38th International Conference on High Energy Physics 3-10 August 2016

Chicago, USA

* Speaker. 


\section{An introduction of KEK and our goals of outreach}

KEK[W] is one of the world's major particle physics research organizations located in Japan. It has two reasearch site : Tsukuba site and Tokai site. Both sites have Japanese cutting-edge accelerators respectively; electron - positoron colliding machine called SuperKEKB at Tsukuba site and high intensity proton machine called J-PARC at Tokai site.

Our mission in research is to unveil mysteries of the Universe, shedding a light to expand the knowledge of human beings. However, sharing these knowledge is also as important as accomplishing our missions. For that purpose, we set our goals of outreach as (1) to give an opportunity "to become a scientist" to young students and nurture future human resource. (2) to share a delight of science, especially particle physics, among general public. (3) to brew a place where we can chit-chat about science

In following three education \& outreach programs among such activities at KEK are described. We would like to introduce these programs and discuss how to make physics, especially high energy physics, more popular in Japan.

\section{B-Lab : An open data analysis program to search for new particles}

B-Lab[] is an open data analysis program started by the Belle experiment group in 2004, which is a first trial for such an open data program in the field of high energy physics. The main target of the program is high school students, but anyone who made a registration can participate in the program.

In the B-Lab program, we provide $1.3 \mathrm{fb}^{-1}$ of the real experimental data taken by the Belle experiment. The data is provided in ROOT format, in the combination of 4-momentum, charge $(0, \pm 1)$ and particle identification $(\pi, K, e, \mu, \gamma)$.

We also provide textbooks and examples of analysis codes, which use ROOT and C++. The textbook describes the introduction of the elementary particle physics, reconstruction of particles and how to run these example codes on ROOT. There are a few exercises to reconstruct a particle from two body decays, where participants are requested to modify the example source codes. Thorough these exercises, participants start searching other reconstruction combinations on their own.

\section{Give an opportunity "to become a scientist"}

Fig.W shows how B-Lab works and stay in touch with participants. (1) First, you send an information needed for registration. The application form is on the B-Lab webpage. (2) After the registration, you can start downloading Belle's data. (3) Once downloaded, analyze data on your own. (4) If you found unknown peaks in your analysis, send an e-mail to the Belle researcher. (5) Then the researcher investigates and gives back feedbacks on what you have found.

Approximately 1400 people have participated in this program. Some participants worked really hard and continued their search at home. And there are continuous registration from certain schools as club activity. Although there were no new particle found so far, a group of high school girls found rare particles $\left(D_{s}\right.$ and $\left.\Lambda_{c}\right)$. Also another group of students presented their work and awarded a prize in a physics contest. 


\section{How B-Lab works}

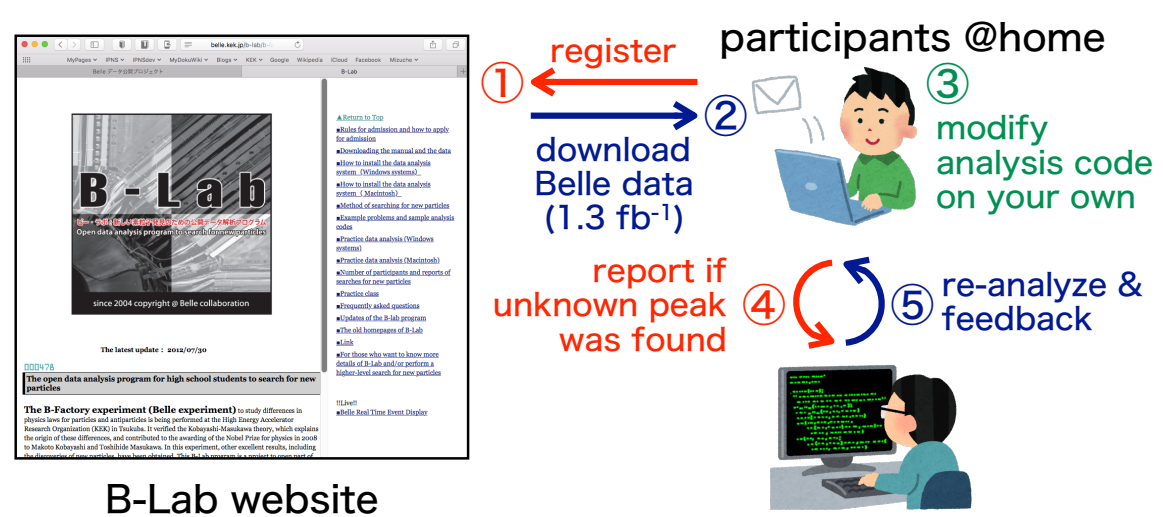

\section{Belle researcher @KEK IPNS}

Figure 1: How B-Lab works between general public and the Belle experiment group

\section{KEK Caravan : A researcher in their alma mater}

KEK caravan[3] is a Japan-wide science masterclass started in June 2010. KEK employees give lecture according to a request from schools and organisations. We send speakers from Hokkaido, the northest prefecture in Japan, to Okinawa, the southest, upon requrest. The application form is on the web.

Fig.[ shows the statistics of the KEK caravan. The histograms on the left shows the number of requests per year and as you can see there are about 60 requests per year. The pie chart on the left shows the distribution of audiences in last year. The total audience was 28,049 and about $79 \%$ were shool students.

\section{Share a delight of science through KEK Caravan}

The key feature of KEK caravan is that a speaker is selected based on their old school, neaby school or hometown where the request came from. We believe that the talk given by home-related researcher would strongly encourage students who wants to be a researcher. 


\section{Number of requests per year}

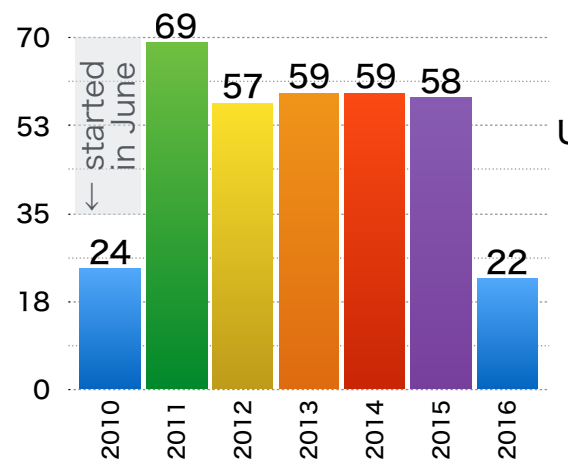

Audiences

(Total : 28049)

Teacher

$2 \%$

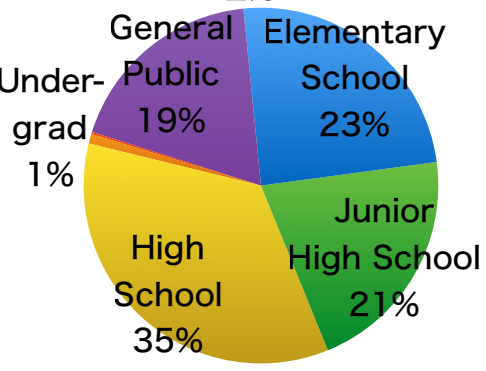

Figure 2: The statistics of the KEK caravan. (Left) A number of requests per year since we started. (Right) A distribution of audiences in last year.

\section{KEK Science Cafe : A science chit-chat on a street corner}

KEK Science Cafe [团] is a weekly science cafe for residents in Tsukuba city, started in November 2015. It is held every friday night at a community space near the Tsukuba station.

Every month we select a topic and a speaker. The topic is selected based on what we research, and the speaker is selected from young researcher; such as $\mathrm{PhD}$ student, posdoc, or research associate.

The number of audiences is about 20 to 30 at a time and the majority of the audiences is students. Since the cafe starts at $7 \mathrm{pm}$, it was totally unexpected to have so many teenagers.

\section{Brew a place where we can chit-chat about science}

The golden rule for the KEK science cafe is just keep up regulary. We strongly believe that if there were a place, people would gather. Unfortunately, there aren't any place in Japan where we can chit-chat science casually and regulary. KEK science cafe would be the first place to provide such a singularity. 


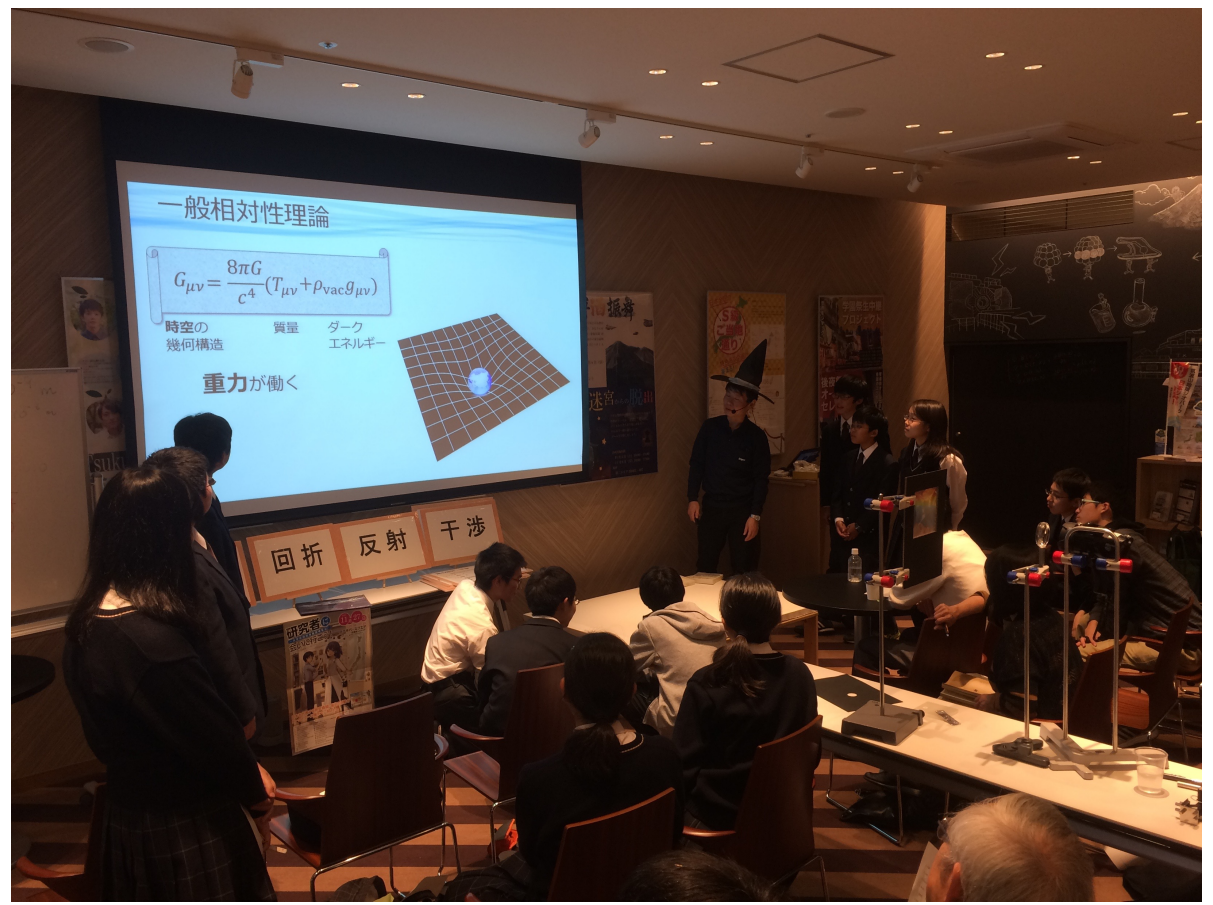

Figure 3: KEK Science Cafe

\section{Summary}

We introduced three education \& outreach programs at KEK : B-Lab, KEK caravan, and KEK science cafe. B-Lab is an open data analysis program mainly for students that gives taste of how to become a scientist. KEK caravan is a Japan-wide masterclass that delivers delight of science to general public. And newly started KEK science cafe is serving as a science chit-chat place for local residents.

\section{References}

[1] KEK Webpage : https://www.kek.jp/en/

[2] B-Lab Webpage : http://belle.kek.jp/b-lab/b-lab-english/

[3] KEK Caravan Webpage : https://www2.kek.jp/caravan/

[4] KEK Science Cafe Webpage : https://www.kek.jp/ja/PublicRelations/Events/ScienceCafe/ 